\title{
Deleterious effect of proton pump inhibitors on the disease course of cirrhosis
}

\author{
Tamás Janka ${ }^{a}$, Tamás Tornai $^{\mathrm{a}}$, Brigitta Borbélyc ${ }^{\mathrm{C}}$, Dávid Tornai ${ }^{\mathrm{b}}$, István Altorjaya ${ }^{\mathrm{a}}$, Mária Papp ${ }^{\mathrm{a},{ }^{\star}}$ and \\ Zsuzsanna Vitális ${ }^{a, *}$
}

\begin{abstract}
Objectives Proton pump inhibitors(PPIs) are widely prescribed to patients with liver cirrhosis. We hypothesized that longstanding PPI use is associated with spontaneous bacterial peritonitis(SBP) and accelerated development of disease-specific complications and liver-related death.

Methods A 5-year follow-up observational cohort study assessed the impact of long-standing PPI use on the clinical course of cirrhosis in a large referral patient cohort. Three hundred fifty patients with cirrhosis (alcohol:69.1\%, Child-Pugh stage A/B/C:206/108/36) were assigned to two groups: regular PPI users $(n=196)$ and nonusers $(n=154)$. Occurrence of SBP, decompensation events (ascites, hepatic encephalopathy and variceal bleeding), and liver-related death were assessed.

Results Regular PPI use was associated with an increased cumulative probability of SBP compared to nonusers [55\% vs. $24.8 \%$, hazard ratio(HR):4.25; $P=0.05]$, in patients without previous SBP episode $(n=84)$. A similar association was found between regular PPI use and decompensation events. The risk of the development of a first decompensation was higher in regular PPI users compared with nonusers, in patients with compensated clinical stage at enrollment (HR: 2.81, $P=0.008, n=146)$. The risk of liver-related death was also significantly increased among regular $P P I$ users $(P<0.001)$. In multivariate Cox-regression analysis, regular PPI use (HR:2.81, $P=0.003)$ and MELD score (HR:1.21, $P<0.001)$ was an independent predictor of mortality.

Conclusion In the present follow-up cohort study, long-term PPI use was associated with the development of SBP and a progressive disease course in patients with cirrhosis that may have been caused by enhanced pathologic bacterial translocation, accelerated development of bacterial translocation-dependent disease-specific complications, and liver-related death. Eur J Gastroenterol Hepatol XXX: 00-00

Copyright () 2019 The Author(s). Published by Wolters Kluwer Health, Inc.
\end{abstract}

\section{Introduction}

The recent identification of specific pathophysiological processes in cirrhosis shows the importance of the possibility of bacterial infections in the prognosis of the disease [1]. Bacterial infections are common causes of morbidity and mortality in the cirrhotic patient population. Disruption of gut barrier function, small bowel bacterial overgrowth (SIBO), dysmotility, and compromised immune defense

\footnotetext{
European Journal of Gastroenterology \& Hepatology 2019, XXX:00-00

Keywords: bacterial translocation, diseases progression, mortality, proton pump inhibitors, spontaneous bacterial peritonitis

aDepartment of Gastroenterology, Faculty of Medicine, Institute of Internal Medicine, University of Debrecen, Kálmán Laki Doctoral School of Biomedical

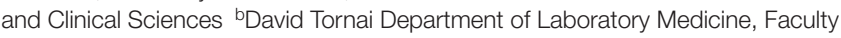
of Medicine Debrecen, University of Debrecen and ${ }^{\mathrm{C} B r i g i t t a}$ Borbely Kenézy Gyula County Hospital, Teaching Hospital of the Medical University of Debrecen, Debrecen, Hungary

Correspondence to Tamás Janka, MD, Department of Internal Medicine, Division of Gastroenterology, Faculty of Medicine, University of Debrecen, Nagyerdei krt. 98, H-4032 Debrecen, Hungary

Tel/fax: +36 52255 152; e-mail: janka.tamas@med.unideb.hu

*Dr. Maria Papp and Dr. Zsuzsanna Vitalis contributed equally to the writing of this article.

\section{Received 26 February 2019 Accepted 23 May 2019}

This is an open-access article distributed under the terms of the Creative Commons Attribution-Non Commercial-No Derivatives License 4.0 (CCBYNCND), where it is permissible to download and share the work provided it is properly cited. The work cannot be changed in any way or used commercially without permission from the journal.
}

each increase the chances of pathological bacterial translocation [2]. Bacteria and bacterial products in the systemic circulation can decompensate hepatic status and induce elevation of portal pressure, creating complications - including variceal bleeding, hepatic encephalopathy, and renal failure - and are responsible for enhanced disease progression. Spontaneous bacterial peritonitis $(\mathrm{SBP})$ is the most common infection in cirrhosis and has a great probability of recurrence. Preventive measures are important. Prophylactic antibiotic treatment can decrease recurrence [3], but it induces bacterial resistance [4]. Novel, nonantibiotic-based prophylaxes are highly needed but still in an experimental phase.

As proton pump inhibitors (PPIs) are presumed to have insignificant side effects, although recent research suggests otherwise, they are commonly used without appropriate indications [5]. They are highly effective in the treatment of peptic ulcer disease, especially in bleeding episodes, because the alkaline shift in gastric $\mathrm{pH}$ has a beneficial effect on the stabilization of blood clot [6,7]. PPIs are also widely used in the treatment and prevention of variceal bleeding in cirrhosis.

Patients with cirrhosis often prescribed PPIs, even though the indication for their use is not always straightforward. It is possible that these patients have higher incidence of gastroesophageal reflux (GERD), which causes symptoms, or that doctors use PPIs for prophylactic reasons. Previous studies postulated that ascites accumulation in patients with cirrhosis may result in 
GERD [8]. Large and endoscopically treated varices may alter the esophageal motility and disturb the esophageal sphincter function, which might also provoke GERD $[9,10]$. By damaging the otherwise thin wall of varices, acidic irritation may induce their rupture. This suggests that GERD might be a provoking factor of variceal bleeding and that PPIs may have a prophylactic effect. Despite this promising hypothesis, evidence for the protective role of PPIs in variceal bleeding is poor. Several clinical trials demonstrated no improvement in the risk of portal hypertension-related bleeding with PPI treatment $[11,12]$. Another problem is that although in some studies GERD was more common in patients with cirrhosis as compared with a healthy population, the low sample size of these studies makes their results highly questionable $[13,14]$. Regardless of these uncertainties, PPIs are frequently prescribed for patients with cirrhosis. This not only increases economic costs in daily clinical practice but, according to an increasing body of research, can also lead to unfavorable side effects, including osteoporosis, pneumonia [15], SIBO, enteric infections, and Clostridium difficile colitis [16]. The association of PPI use with the occurrence of SBP in cirrhotic patients is the most extensively evaluated adverse outcome. Some studies found an association [17-21] and some studies did not find one [22-24]. Three out of four meta-analyses found an association between PPI use and development of SBP [25-27]. A fourth meta-analysis concluded that the association has little clinical relevance. Recent high-quality studies with a large sample size did not find an association [28]. The problem with evaluating these studies is that they differ in study design, sample size, inclusion criteria, and the inconsistency in specifying the exact duration of PPI use.

SBP can be a sign of increased bacterial translocation. Previous research has concentrated only on the diagnosis of SBP. We hypothesized that long-term PPI use is linked to enhanced bacterial translocation-related complications in general. If this is right, PPI use would be associated not only with SBP but also with enhanced disease progression, for example, the appearance of the first decompensation event or liver-related death. For this reason, we evaluated more general effects of PPIs in patients with decompensated and compensated liver disease.

The aim of the present follow-up observational study of PPI use in a large referral patient cohort with cirrhosis was to determine if long-term PPI administration constitutes a risk for: (1) SBP; (2) a progressive disease course, namely, the advent of decompensation events (development of ascites, hepatic encephalopathy, and variceal bleeding); and (3) mortality.

\section{Material and methods}

\section{Patient population}

We conducted a cohort study of 350 adult patients with established diagnosis of cirrhosis of different etiologies in a referral hepatology center (Division of Gastroenterology, Department of Internal Medicine, Clinical Center, University of Debrecen, Hungary).

The patients in the study group were a subgroup of 404 patients with cirrhosis recruited between 1 May 2006 and 31 December 2010 [29]. We selected 350 patients who were stable, that is, had no signs of acute decompensation in regular or extraordinary follow-up visits and did not meet three exclusion criteria: (1) the patient or legal surrogate declined to participate in this study and did not sign the informed consent, (2) the patient was sent for a single specialist consultation only and was followed up regularly elsewhere, or (3) the patient had a follow-up shorter than 3 months.

Diagnosis of cirrhosis was based on clinical, biochemical, imaging, and, when available, histological data. Routine laboratory data and detailed clinical phenotypes were captured at inclusion. Clinical data were determined by in-depth review of detailed medical records. Age at diagnosis, etiology, presence of hepatocellular carcinoma (HCC), presence of esophageal varices, history of previous acute decompensation episodes, and cirrhosis-related medication were retrospectively analyzed for the period prior to the observational follow-up study. At enrollment into the study, disease severity was assessed by liver-oriented scores (Child-Pugh and MELD) and the clinical stage of the diseases (compensated/ decompensated) [30] were determined.

The data collection, comprising clinical variables and outcomes, and also medication was collected prospectively.

\section{Phenotypical characterization of patients during follow-up}

Attending gastroenterologists registered the date and type of acute decompensation episodes during previous hospital admissions of the patients in the study. Acute decompensation was defined by acute development of large ascites (grade II/III), acute hepatic encephalopathy, acute variceal bleeding, or presence of systemic bacterial infection, including SBP. Diagnosis of SBP was based on ascitic fluid polymorphonuclear cell count exceeding $250 / \mathrm{mm}^{3}$, with or without positive ascites culture, following the AASLD Practice Guidelines of 2004 [31]. The follow-up period lasted 5 years, or until death or loss of follow-up. One hundred forty-seven $(42 \%)$ patients died during follow-up. The median time to death was 575 days [interquartile range (IQR): 286-885]. In the 203 patients alive at the end of the follow-up, the median follow-up lasted 1155 days (IQR: 646-1741). Collected data were transferred to and stored in a database. At the end of the study period, 31 December 2013, attending gastroenterologists checked medical records registered during regular and extraordinary outpatient follow-up visits and inpatient stays to identify long-standing PPI use.

The study cohort $(N=350)$ was divided into two groups: regular PPI users $(n=196)$ and nonusers $(n=154)$. Regular PPI users were defined as taking the drug for at least $80 \%$ of the follow-up period; nonusers were defined as not taking PPI at all during the study. Fifty-four of enrolled patients were excluded at the end because of episodic or uncertain PPI use. Clinical characteristics of patients at inclusion are presented in Table 1. In Hungary, a regular outpatient follow-up visit for patients with decompensated cirrhosis is usually scheduled every 3 months after hospitalization for acute decompensation at a specialized gastroenterology center. Follow-ups might be scheduled between every 1-3 months if dictated by disease severity or the presence of disease-specific complications, 


\begin{tabular}{lc}
\hline Table 1. Clinical characteristics of patients with cirrhosis & \\
\hline & \\
Number & Total \\
\hline Gender (male/female) & 350 \\
Age (years) (median, IQR) & $188 / 162$ \\
Alcoholic etiology & $56(50-64)$ \\
Child-Pugh stage, $n$ (\%) & $242(69.1)$ \\
A & \\
B & $206(58.9)$ \\
C & $108(30.9)$ \\
MELD score a & $36(10.3)$ \\
Decompensated stage, $n$ (\%) & $11.5(8-16)$ \\
Comorbidity, $n$ (\%) & $204(58.3)$ \\
HCC, $n$ (\%) & $171(48.9)$ \\
Ascites, $n$ (\%) & $33(9.4)$ \\
Prior variceal bleeding, $n$ (\%) & $113(32.3)$ \\
Prior hepatic encephalopathy, $n(\%)$ & $100(28.6)$ \\
Prior SBP, $n$ (\%) & $19(5.4)$ \\
& $44(12.6)$ \\
\hline P vaus
\end{tabular}

$P$ values were calculated with Mann-Whitney U-test, $\chi^{2}$-test or Fisher's exact test as appropriate.

HCC, hepatocellular carcinoma; IQR, inter quartile range; MELD: Model for end-stage liver disease score; NS: Nonsignificant; PPI, proton pump inhibitor; SBP, spontaneous bacterial peritonitis.

aMedian, IQR (lowest 25\%-highest 25\%).

and for patients with cirrhosis but without a prior episode of acute decompensation follow-ups are scheduled for every 6 months maximum. This follow-up schedule enables reliable tracing of drug-taking habits.

\section{Ethical permission}

The study protocol was approved by the Regional and Institutional Research Ethics Committee of University of Debrecen and the National Scientific and Research Ethics Committee (DEOEC-RKEB/IKEB 5306-9/2011, 3885/2012/EKU [60/PI/2012], 9485-1/2016/EKU ad 167/2016). Patients were informed of the nature of the study and gave written informed consent.

\section{Statistical analysis}

Variables were tested for normality using Shapiro Wilk's W test. Continuous variables were summarized as means (SD) or as medians (interquartile range [IQR], lowest $25 \%$-highest $25 \%$ ) according to their homogeneity. Categorical variables were compared with Fisher's exact test or $\chi^{2}$ test with Yates correction, as appropriate. Continuous variables were compared with Mann-Whitney U test or Kruskal-Wallis H test with Dunn's multiple comparison post-hoc analysis. Paired samples were analyzed by Wilcoxon signed-rank test. Kaplan-Meier analysis was used to calculate the cumulative probability of adverse outcome (SBP, decompensation event, and mortality). Differences in observed probabilities were assessed by the log-rank test. The association between categorical clinical variables or PPI use and adverse disease outcomes during follow-up was assessed by univariate Cox-regression analysis. Multivariate analyses were performed with backward elimination procedure and likelihood ratio test to identify independent predictors. Associations are given as hazard ratio (HR) with $95 \%$ confidence intervals (CIs). For statistical analysis and graphical presentation, the SPSS 22.0 (SPSS, Chicago, Illinois, USA), and GraphPad Prism 6 (San Diego, California, USA) programs were used. A two-sided probability value of $<0.05$ was considered to be statistically significant.

\section{Results}

\section{Study population}

Three hundred fifty patients with cirrhosis were enrolled in the study. At the time of inclusion, $196(56.0 \%)$ were PPI users. The main characteristics of patients are summarized in Table 1 . There were 188 men and 162 women, with a median age of 56 years (range: 50-64) in our study. The main baseline characteristics were as follows: alcoholic liver disease in 242 out of 350 patients $(69.1 \%)$. One hundred seventy-one patients $(48.9 \%)$ had extrahepatic comorbidities. One hundred thirteen $(32.3 \%)$ had ascites at inclusion, while $100(28.6 \%)$ had prior variceal bleeding or $19(5.4 \%)$ had prior hepatic encephalopathy episodes. The distribution of Child-Pugh stage was the following: 58.9, 30.9, and $10.3 \%$ for Child A, B, and C, respectively.

Among patients with ascites at inclusion (113) the PPI user and nonuser patient groups did not differ in gender, age, or presence of comorbidities or HCC, and disease stage, as indicated by median values of MELD score, or distribution of Child-Pugh stage. (Table 2).

One hundred forty-six patients had compensated clinical stage at the time of inclusion (Table 3). Compensated patients had no ascites, variceal bleeding, hepatic encephalopathy, or SBP at inclusion or in their previous history. The PPI user and nonuser patient groups did not differ significantly in gender, age, alcoholic etiology, presence of comorbidities, HCC nor had they advanced disease stage according to MELD score, or distribution of Child-Pugh stage.

In patients with compensated disease stage, the primary endpoint of the study was the development of the first decompensation event (hepatic encephalopathy, variceal bleeding, and ascites). In patients with decompensated disease stage (patients with ascites), the primary endpoint was the development of SBP.

The secondary endpoint was the liver-related death in both patient groups.

\section{Risk factors of spontaneous bacterial peritonitis}

Of all patients in the study with ascites, 31.9\% (36/113) developed at least one SBP episode during the follow-up period. The median time to the development of first SBP episode was 366 days (IQR, 153-969). Prior history of an SBP episode [HR: 2.02 (95\% CI: 1.03-3.94), $P=0.04$ ], but not age, gender, etiology, or severity of the disease were associated with increased risk of SBP development (data not shown). PPI use was associated with an increased cumulative probability of SBP [55.0\% vs. 30\%, HR: 2.76 (95\% CI: $1.2-6.31), P=0.017]$. MELD score did not differ, however, between two patient groups (median, IQR: 16 [13-21] for PPI users vs. 14 [11-18] for PPI nonusers, $P=0.06)$. Further investigation of the association between PPI use and increased risk of SBP development showed that PPI use increased the risk of SBP development only in patients who did not have a previous SBP episode $(n=84)$ (Fig. 1). In this patient group, the cumulative probability of SBP was $55.0 \%$ in PPI users and $24.8 \%$ in nonusers [HR: 4.25 (95\% CI: 1.42-12.67), $P=0.05$ ]. No significant difference was found in patients who had a previous SBP episode $(n=29)[55.7 \%$ vs. $50.0 \%$, HR: $0.82(95 \%$ CI: $0.23-2.93), P=\mathrm{NS}$ ]. 
Table 2. Clinical characteristics of patients with ascites at inclusion

\begin{tabular}{|c|c|c|c|c|}
\hline Number & $\begin{array}{l}\text { Total } \\
113\end{array}$ & $\begin{array}{c}\text { PPI user } \\
74\end{array}$ & $\begin{array}{c}\text { PPI nonuser } \\
39\end{array}$ & $P$ value \\
\hline Gender (male/female) & $69 / 77$ & $48 / 26$ & $19 / 20$ & 0.098 \\
\hline Age (years) ${ }^{a}$ (median, IQR) & $58(52-65)$ & $58(52-65)$ & $56(52-66)$ & 0.962 \\
\hline Alcoholic etiology & $93(82.3)$ & $62(83.8)$ & $31(79.5)$ & 0.571 \\
\hline \multicolumn{5}{|l|}{ Child-Pugh stage, $n$ (\%) } \\
\hline A & $19(16.8)$ & $14(18.9)$ & $5(12.8)$ & 0.057 \\
\hline B & $61(54.0)$ & $34(45.9)$ & 27 (69.2) & \\
\hline C & $33(29.2)$ & $26(35.1)$ & 7 (17.9) & \\
\hline MELD score ${ }^{\mathrm{a}}$ & $15(12-19)$ & $16(13-21)$ & $14(11-18)$ & 0.055 \\
\hline Comorbidity, $n$ (\%) & $57(50.4)$ & $42(56.8)$ & $15(38.5)$ & 0.066 \\
\hline $\mathrm{HCC}, n(\%)$ & $11(9.7)$ & $7(9.5)$ & $4(10.3)$ & 0.892 \\
\hline Prior variceal bleeding, $n(\%)$ & $31(27.4)$ & $25(33.8)$ & $6(15.4)$ & 0.038 \\
\hline Prior hepatic encephalopathy, $n(\%)$ & $11(9.7)$ & $11(14.9)$ & $0(0.0)$ & 0.012 \\
\hline Prior SBP, $n(\%)$ & $28(24.8)$ & $22(29.7)$ & $6(15.4)$ & 0.095 \\
\hline
\end{tabular}

$P$ values were calculated with Mann-Whitney U-test, $\chi^{2}$-test or Fisher's exact test as appropriate.

HCC, hepatocellular carcinoma; IQR, inter quartile range; MELD: Model for end-stage liver disease score; NS: Nonsignificant; PPI, proton pump inhibitor; SBP, spontaneous bacterial peritonitis.

aMedian, IQR (lowest 25\%-highest 25\%).

\begin{tabular}{|c|c|c|c|c|}
\hline Number & $\begin{array}{c}\text { Total } \\
146\end{array}$ & $\begin{array}{l}\text { PPI user } \\
50\end{array}$ & $\begin{array}{c}\text { PPI nonuser } \\
96\end{array}$ & $P$ value \\
\hline Gender (male/female) & 69/77 & $21 / 29$ & $48 / 48$ & 0.360 \\
\hline Age (years) (median, IQR) & $56(49-64)$ & $56.5(49-64)$ & $56(51-64)$ & 0.977 \\
\hline Alcoholic etiology & $74(50.7)$ & $27(54.0)$ & $47(49.0)$ & 0.564 \\
\hline \multicolumn{5}{|l|}{ Child-Pugh stage, $n(\%)$} \\
\hline A & $121(82.9)$ & $38(76.0)$ & $83(86.59)$ & 0.111 \\
\hline B & $25(17.1)$ & $12(24.0)$ & $12(13.5)$ & \\
\hline $\mathrm{C}$ & & & & \\
\hline MELD score ${ }^{a}$ & $9(7-13)$ & $9(7-13)$ & $8(7-11)$ & 0.118 \\
\hline Comorbidity, $n$ (\%) & $71(48.6)$ & $25(50.0)$ & $46(47.9)$ & 0.812 \\
\hline HCC, $n(\%)$ & $17(11.6)$ & $4(8.0)$ & $13(13.5)$ & 0.324 \\
\hline
\end{tabular}

$P$ values were calculated with Mann-Whitney $U$-test, $\chi 2$-test or Fisher's exact test as appropriate.

$\mathrm{CC}$, hepatocellular carcinoma; IQR, inter quartile range; MELD, Model for endstage liver disease score; NS, Nonsignificant; PPI, proton pump inhibitor. aMedian, IQR (lowest 25\%-highest 25\%)

\section{Advent of first decompensation event}

Of patients with compensated clinical stage, $18.5 \%$ (27/146) experienced a decompensation event (ascites, variceal bleeding, or hepatic encephalopathy). The median time to the development of the first decompensation episode was 540 days [IQR, 189-900]. PPI use was associated with an increased cumulative probability of the development of the first decompensation event compared with PPI nonuse $(41.9 \% \pm 8.5$ vs. $17.7 \% \pm 6.4$ with HR: $2.81,95 \%$ CI: $1.31-6.01, P=0.008$ ) (Table 4 and Fig. 2). Sensitivity analysis according to the PPI dose showed that only long-standing double dose PPI therapy (HR: 6.63, 95\% Cl: 2.7-16.3, $P<0.001$ ) and not the standard one (HR: $2.09,95 \% \mathrm{Cl}: 0.85-5.14$, $P=0.106)$ associated to higher risk of decompensation of the disease.

\section{Covariates}

Univariate Cox regression analysis of clinical factors associated with the development of a decompensation event is shown in Table 4. Higher MELD score (HR: 1.21, 95\% CI: $1.10-1.34, P<0.001)$ was significantly associated with the increased risk for the development of the first decompensation event.

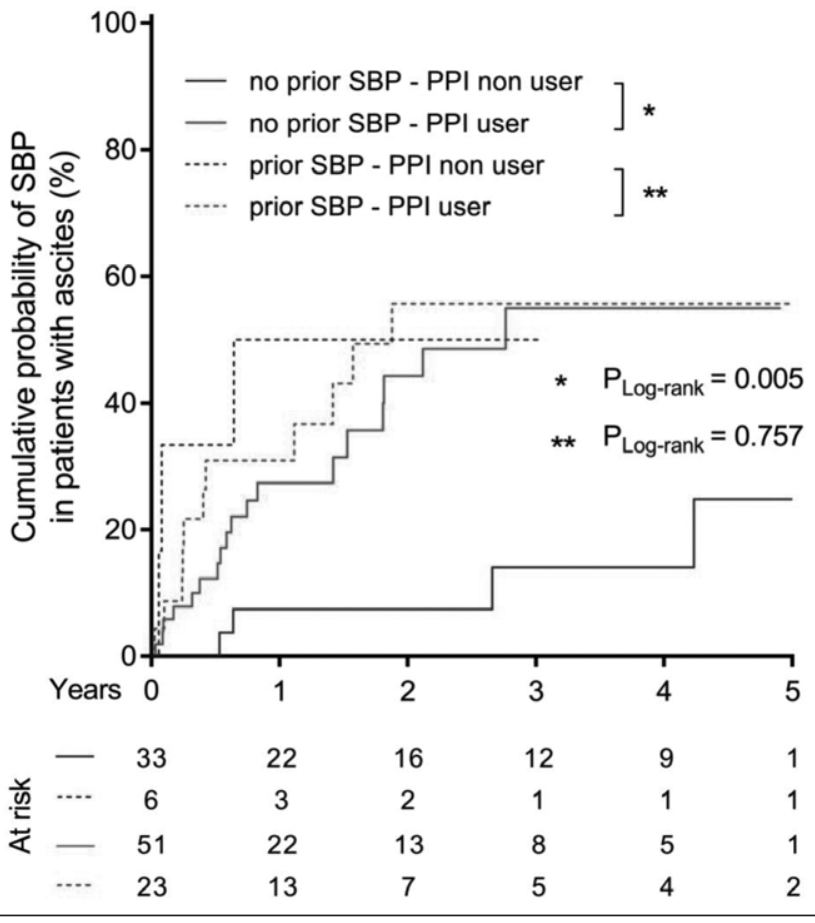

Fig. 1. Development of spontaneous bacterial peritonitis in patients without previous SBP episode according to PPI use. PPI use increased the risk of SBP development in those patients who had not got previous SBP episode. PPI, proton pump inhibitor; SBP, spontaneous bacterial peritonitis.

\section{Multivariate analysis}

Cox regression analysis and the backward elimination procedure, taking the status of PPI use and MELD into account, indicated that PPI use, and higher MELD score were independently associated with the risk of the development of first decompensating event (Table 4).

\section{Mortality}

In the total cohort, liver-related mortality occurred in 147 $(42.0 \%)$ patients. Kaplan-Meier survival analysis demonstrated a significantly worse survival in patients with advanced disease, according to the presence of decompensated clinical stage $(P<0.001)$ or higher MELD score $(P<0.001)$, age $(P=0.026)$, comorbidity $(P=0.007)$, 
and alcoholic etiology $(P=0.005)$. Long-standing PPI use $(P<0.001)$ was also associated with a lower survival rate (Table 5 and Fig. 3). PPI use (HR: 2.01, 95\% CI: $1.38-$ 2.93, $P<0.001$ ), and clinical stage (HR: $1.66,95 \%$ CI: $1.12-2.45, P=0.011$ ) and MELD score (HR: 1.12, 95\% CI: $1.08-1.55, P<0.001)$ remained independent predictors of mortality in multivariate analysis, as well (Table 5).

Further analyzing the mortality risk between groups of patients with different disease severity, we revealed that the PPI use was only associated with higher risk of mortality in the compensated patient group (HR: 2.81, 95\% Cl: $1.43-5.51, P=0.003)$, but not in the decompensated

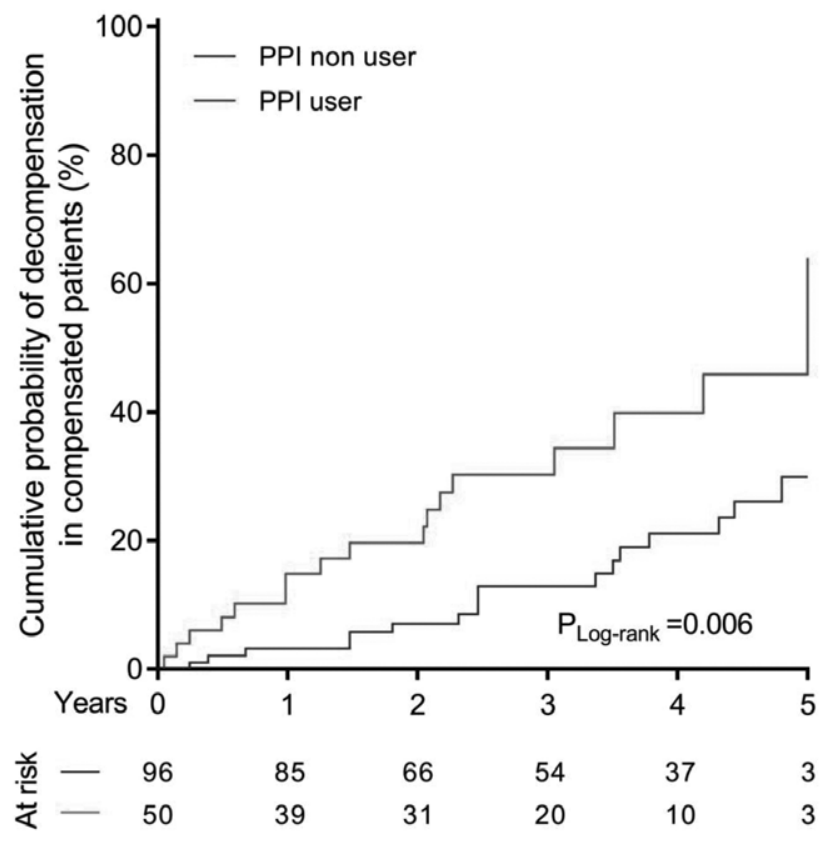

Fig. 2. Development of first decompensation event in patients with compensated clinical stage according to PPI use. PPI use was associated with an increased cumulative probability of the development of first decompensation event compared to PPI nonuse. PPI, proton pump inhibitor. one (HR: $1.42,95 \% \mathrm{Cl}: 0.89-2.26, P=1.37$ ). These findings, however, were independent of PPI dosage in both the compensated (HR: 2.73, 95\% Cl: 1.31-5.69, $P=0.008$ and HR: 4.28, 95\% Cl: $1.88-9.73, P=0.001)$ and decompensated patient group (HR: 1.64, 95\% Cl: 0.99-2.72, $P=0.54$ and HR: $1.58,95 \% \mathrm{Cl}: 0.95-2.63, P=0.81)$.

\section{Discussion}

Most previous clinical studies assessing the impact of PPI use in cirrhosis addressed patients with an advanced disease stage. None included patients with a compensated disease stage. These studies tell us primarily about the development of SBP in ascitic patients and very little about the long-term effects of PPI use or disease-specific complications beyond SBP among patients with cirrhosis [32]. This study addresses the gap in our knowledge by comprehensively assessing the significance of long-term PPI use in a large cohort of patients on the entire severity spectrum of cirrhosis, with special emphasis on the progressive disease course.

Previous studies [17-28] evaluating the impact of PPI use on SBP development have yielded inconsistent results. Most of the inconsistencies can be attributed to differences in study design, sample size, and inclusion criteria. Definitions of PPI use were often vague and did not specify the exact duration of PPI use. To address the possibility that the unfavorable effects of PPI use need time to develop, this study used a long follow-up time (median of 1155 days). To ensure clarity of definition of PPI use, we followed rigorous criteria to divide subjects into PPI user and nonuser patient groups and excluded patients with episodic or uncertain PPI use.

In the present study, long-term PPI use was found to be significantly associated with increased risk of SBP development in patients with ascites, which is only one consequence of PPI-provoked bacterial translocation. According to the most widely accepted hypothesis for the association of PPI use and SBP, decreased gastric acid

Table 4. Univariate and multivariate Cox-regression analysis for the association of proton pump inhibitor use and clinical factors with the cumulative probability of decompensation patients with compensated clinical stage

\begin{tabular}{|c|c|c|c|c|c|c|c|c|}
\hline & \multicolumn{3}{|c|}{ Development of decompensation } & \multirow[b]{2}{*}{$P$ value ${ }^{a}$} & \multicolumn{2}{|c|}{ Univariate analysis } & \multicolumn{2}{|c|}{ Mulivariate analysis } \\
\hline & $n$ of subjects & $n$ of events & $\begin{array}{c}\text { Cumulative probability } \\
\text { of event } \%\end{array}$ & & $\mathrm{HR}(95 \% \mathrm{Cl})$ & $P$ value & $\mathrm{HR}(95 \% \mathrm{Cl})$ & $P$ value \\
\hline Compensated stage & 146 & 27 & 25.6 & - & & & & \\
\hline \multicolumn{9}{|l|}{ Age (years) } \\
\hline$<65$ & 113 & 20 & 23.2 & & & & & \\
\hline$>65$ & 33 & 7 & 35.2 & 0.398 & $1.48(0.62-3.52)$ & 0.376 & & \\
\hline \multicolumn{9}{|l|}{ Gender } \\
\hline Male & 70 & 15 & 29.4 & & & & & \\
\hline Female & 76 & 12 & 21.8 & 0.304 & $1.49(0.7-3.18)$ & 0.306 & & \\
\hline \multicolumn{9}{|l|}{ Comorbidity } \\
\hline Absent & 75 & 11 & 19.8 & & & & & \\
\hline Present & 71 & 16 & 32.3 & 0.162 & $1.69(0.78-3.65)$ & 0.18 & & \\
\hline \multicolumn{9}{|l|}{ Etiology } \\
\hline Other & 71 & 8 & 18.9 & & & & & \\
\hline Alcohol & 75 & 19 & 31.8 & 0.042 & $2.30(1.00-5.25)$ & 0.049 & $1.55(0.66-3.67)$ & 0.31 \\
\hline \multicolumn{9}{|c|}{ MELD score (per 1 point increase) } \\
\hline NA & NA & & NA & & $1.21(1.10-1.34)$ & $<0.001$ & $1.21(1.08-1.35)$ & 0.001 \\
\hline \multicolumn{9}{|l|}{ PPI use } \\
\hline No & 96 & 12 & 17.7 & & & & & \\
\hline Yes & 50 & 15 & 41.9 & 0.004 & $2.81(1.31-6.01)$ & 0.008 & $2.58(1.20-5.55)$ & 0.015 \\
\hline
\end{tabular}

$\mathrm{Cl}$, confidence interval; $\mathrm{HR}$, hazard ratio; NA, not applicable; PPI, proton pump inhibitor.

${ }^{a} P$ values were calculated by the log-rank test. 
Table 5. Univariate and multivariate Cox-regression analysis for the association of proton pump inhibitor use and clinical factors with the liver-related mortality

\begin{tabular}{|c|c|c|c|c|c|c|c|c|}
\hline & \multicolumn{3}{|c|}{ Mortality event } & \multirow[b]{2}{*}{$P$ value $^{a}$} & \multicolumn{2}{|c|}{ Univariate analysis } & \multicolumn{2}{|c|}{ Mulivariate analysis } \\
\hline & $n$ of subjects & $n$ of events & $\begin{array}{c}\text { Cumulative probability } \\
\text { of event } \%\end{array}$ & & HR (95\% Cl) & $P$ value & HR $(95 \% \mathrm{Cl})$ & $P$ value \\
\hline Total cohort & 350 & 147 & 42 & - & & & & \\
\hline \multicolumn{9}{|l|}{ Age (years) } \\
\hline$<65$ & 296 & 104 & 53.2 & & & & & \\
\hline$>65$ & 81 & 43 & 79.2 & 0.026 & $1.49(1.05-2.13)$ & 0.027 & $1.40(0.98-2.01)$ & 0.069 \\
\hline \multicolumn{9}{|l|}{ Gender } \\
\hline Male & 188 & 80 & 57.8 & & & & & \\
\hline Female & 162 & 67 & 61.4 & 0.647 & $0.93(0.67-1.28)$ & 0.647 & & \\
\hline \multicolumn{9}{|l|}{ Comorbidity } \\
\hline Absent & 171 & 59 & 50.3 & & & & & \\
\hline Present & 179 & 88 & 68.2 & 0.007 & $1.57(1.13-2.18)$ & 0.008 & $1.32(0.93-1.85)$ & 0.152 \\
\hline \multicolumn{9}{|l|}{ Etiology } \\
\hline Other & 108 & 31 & 39.6 & & & & & \\
\hline Alcohol & 242 & 116 & 67.5 & 0.005 & $1.76(1.18-2.62)$ & 0.005 & $1.12(0.729-1.726)$ & 0.6 \\
\hline MELD score (per 1 point increase) & & & & $<0.001$ & $1.14(1.1-1.17)$ & $<0.001$ & $1.12(1.08-1.55)$ & $<0.001$ \\
\hline \multicolumn{9}{|l|}{ Clinical stage } \\
\hline Comp. & 163 & 46 & 42.8 & & & & & \\
\hline Decomp. & 187 & 101 & 71.6 & $<0.001$ & $2.52(1.78-3.58)$ & $<0.001$ & $1.66(1.12-2.45)$ & 0.011 \\
\hline \multicolumn{9}{|l|}{ PPI use } \\
\hline No & 154 & 39 & 33.9 & & & & & \\
\hline Yes & 196 & 108 & 75.1 & $<0.001$ & $2.63(1.82-3.79)$ & $<0.001$ & $2.01(1.38-2.93)$ & $<0.001$ \\
\hline
\end{tabular}

$\mathrm{Cl}$, confidence interval; Comp, clinically compensated disease stage; Decomp, clinically decompensated disease stage; HR, hazard ratio; PPI, proton pump inhibitor.

a $P$ values were calculated by the log-rank test.

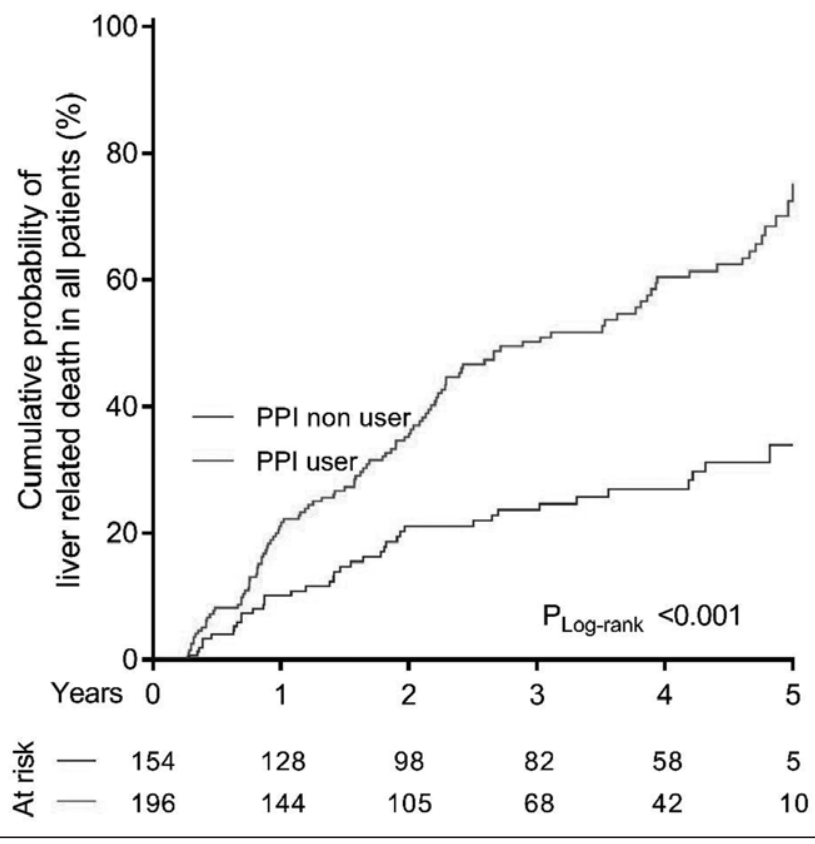

Fig. 3. Liver-related mortality according to PPI use. PPI use $(P<0.001)$ is associated with worse survival. PPI, proton pump inhibitor.

production enhances the development of small intestinal bacterial overgrowth (SIBO). Gastric acid has a strong bactericidal effect, which can decontaminate the stomach and proximal small intestine. PPI therapy may interfere with this first line of immune defense against orally entering pathogens. PPI has also been shown to slow gastrointestinal motility [33], delay gastric emptying [34], and decrease gastric mucus viscosity [35], all of which might have direct effects on gut microflora and the survival of enteric pathogens that may lead to SIBO. Some of the studies could not confirm the more common SIBO in PPI users [36], but the above-mentioned PPI effects and the fact that SIBO is commonly developed in the absence of gastric acid after a total gastrectomy [37] support this theory. A recent subgroup meta-analysis of recently published large-scale meta-analysis unequivocally supports this idea [38]. Studies that employed either a direct test (culture of small bowel aspirates) or an indirect test of SIBO (glucose hydrogen breath tests) were included in the meta-analysis. Direct tests confirmed the association between PPI use and SIBO [39], indirect tests could not [36]. The analysis concluded that use of PPI moderately increases the risk of SIBO with the pooled odds ratio of 1.71 (95\% CI: 1.20 2.43) [38].

We must suppose that SIBO is not the only factor that provokes SBP. PPI therapy does not only provoke SIBO but it can also have other undesirable effects, for example, it can alter the composition and function of the intestinal microflora [40]. Interestingly, Wallace et al. [41] reported that PPI might exacerbate nonsteroidal anti-inflammatory drug-induced small bowel injury, which they explained as a consequence of dysbiosis.

In addition to its acid-suppressive effect, PPI can also weaken the innate immune response by impairing neutrophil function. As appropriate $\mathrm{H}^{+}$-pump activity is necessary for the rapid release of toxic reactive oxygen species and interleukin- 8 production, inhibition of the $\mathrm{H}^{+}$-pump due to acid suppression can decrease both the function and recruitment of inflammatory cells reducing bactericidal activity [42]. These mechanisms elucidate the role of PPI in the development of pneumonia and other serious healthcare-associated and hospital-acquired infections [43]. SIBO, dysbiosis, and altered immune function all enhance bacterial translocation.

As PPI is considered to be well tolerated, its dose is usually not adjusted to liver function. It is known that the half-life of PPI is increased in patients with impaired liver function. As a result, prolonged high serum concentration of the drug potentiates acid suppression and may increase 
PPI associated side effects [44]. Immune suppressive effects can be more harmful in patients with cirrhosis known to be immunocompromised with a multifactorial state of systemic immune dysfunction [45]. The unnecessarily high dose of the drug further enhances negative effects of PPI.

We found, for the first time, that PPI use increased the risk of SBP development only for patients who did not have a previous SBP episode. Initiation of antibiotic treatment as a secondary prophylaxis might counteract the enhanced bacterial translocation induced by PPI treatment, and so make subsequent episodes less likely.

Habits of PPI use in cirrhosis show that the drug is more commonly prescribed for patients with advanced disease stage [32] that was also found in our study. Abdominal discomfort is frequent in this stage of the disease due to the accumulation of large volume of ascites that can be mistakenly treated with PPI. Increased frequency of gastroesophageal reflux in this population is a matter of debate.

Pathological bacterial translocation is associated with clinically relevant complications in cirrhosis beyond SBP [46].

Our study is the first to consider the effect of long-term PPI use on the development of a range of bacterial translocation-related diseases. We hypothesized that if long-term PPI use was linked to bacterial translocation, it would be associated with enhanced disease progression, for example, the appearance of the first decompensation event or liver-related death. Our results confirmed this hypothesis and are in accord with the recent finding of Llorente et al. [47] that PPI use increased the risk of alcohol-dependent patients developing alcoholic liver disease. They proposed a background mechanism based on data from mouse models in which gastric acid suppression increases intestinal overgrowth of Enterococcus. Translocation of this type of gut bacteria into the liver via the portal vein results in the binding of Enterococcus to the toll-like receptor 2 of hepatic Kupffer cells and secretion of IL1B. IL1B contributes to ethanol-induced liver inflammation and hepatocyte damage.

The worst outcome, mortality, was also evaluated. The effect of PPI on mortality has been assessed in only one previous study [32]. It found that PPI use was an independent predictor of mortality (HR: 2.330 [95\% CI, 1.264-4.296], $P=0.007)$. We also found an increased hazard of death related to PPI use (HR: 2.01 [95\% CI, 1.38-2.93], $P<0.001$ ).

In summary, we were able to confirm previous findings that long-term PPI use increased the risk of SBP, and we found that PPI use was associated with the development of clinical decompensation and liver-related death during follow-up. These results support our hypothesis that $\mathrm{SBP}$ is only one consequence of enhanced bacterial translocation provoked by PPIs, which warrants a cautious approach to prescribe PPIs to patients with cirrhosis.

\section{Acknowledgements}

Core tip: Findings have been inconsistent regarding the association of PPI use with the incidence of SBP. Increased occurrence of SBP in PPI users was previously attributed to the induction of SIBO. SIBO is an important element of pathologic bacterial translocation; however, PPI has several other effects that could also increase bacterial translocation beyond SIBO. Bacterial translocation has been established as an important risk factor of cirrhosis progressing beyond SBP. The present study found that long-term PPI administration was associated with the progressive disease course in cirrhosis, namely the advent of decompensation events and liver-related mortality.

Z.V., M.P., and A.I. designed research; Z.V., T.J., M.P., T.T., and D.T. performed research;Z.V., M.P., and T.T. analyzed data; Z.V., M.P., and T.J. wrote the paper.

All study participants, or their legal guardian, provided informed written consent prior to study enrollment.

This work was supported by the Research Grant of National Research Development and Innovation Office (K115818/2015/1), GINO P-2.3.2-15-2016-00048 and EFOP -3.6.1-16-2016-00022 and 3.6.2-16-2017-00006 projects. EFOP projects are co-financed by the European Union and the European Social Fund. M.P. was supported by the János Bólyai Research Scholarship of Hungarian Academy of Sciences (BO/00232/17/5) and New National Excellence Program of the Ministry of Human Capacities (ÚNKP-18-4).

\section{Conflicts of interest}

There are no conflicts of interest.

\section{References}

1 Arvaniti V, D’Amico G, Fede G, Manousou P, Tsochatzis E, Pleguezuelo $M$, Burroughs AK. Infections in patients with cirrhosis increase mortality four-fold and should be used in determining prognosis. Gastroenterology 2010; 139:1246.e1-1256.e1.

2 Bellot P, Francés R, Such J. Pathological bacterial translocation in cirrhosis: pathophysiology, diagnosis and clinical implications. Liver Int 2013; 33:31-39.

3 Saab S, Hernandez JC, Chi AC, Tong MJ. Oral antibiotic prophylaxis reduces spontaneous bacterial peritonitis occurrence and improves short-term survival in cirrhosis: a meta-analysis. Am J Gastroenterol 2009; 104:993-1001; quiz 1002.

4 Tandon P, Delisle A, Topal JE, Garcia-Tsao G. High prevalence of antibiotic-resistant bacterial infections among patients with cirrhosis at a US liver center. Clin Gastroenterol Hepatol 2012; 10:1291-1298.

5 Kalaitzakis E, Björnsson E. Inadequate use of proton-pump inhibitors in patients with liver cirrhosis. Eur J Gastroenterol Hepatol 2008; 20:512-518.

6 Green FW Jr, Kaplan MM, Curtis LE, Levine PH. Effect of acid and pepsin on blood coagulation and platelet aggregation. A possible contributor prolonged gastroduodenal mucosal hemorrhage. Gastroenterology 1978; 74:38-43.

7 Low J, Dodds AJ, Biggs JC. Fibrinolytic activity of gastroduodenal secretions-a possible role in upper gastrointestinal haemorrhage. Thromb Res 1980; 17:819-830.

8 Li B, Zhang B, Ma JW, Li P, Li L, Song YM, Ding HG. High prevalence of reflux esophagitis among upper endoscopies in Chinese patients with chronic liver diseases. BMC Gastroenterol 2010; 10:54.

9 Passaretti S, Mazzotti G, de Franchis R, Cipolla M, Testoni PA, Tittobello A. Esophageal motility in cirrhotics with and without esophageal varices. Scand J Gastroenterol 1989; 24:334-338.

10 Reilly JJ Jr, Schade RR, Van Thiel DS. Esophageal function after injection sclerotherapy: pathogenesis of esophageal stricture. Am J Surg 1984; 147:85-88.

11 Garcia-Saenz-de-Sicilia M, Sanchez-Avila F, Chavez-Tapia N-C, Lopez-Arce G, Garcia-Osogobio S, Ruiz-Cordero R, et al. PPls are not associated with a lower incidence of portal-hypertension-related bleeding in cirrhosis. World J Gastroenterol 2010; 16:5869-5873. 
12 Lo EA, Wilby KJ, Ensom MH. Use of proton pump inhibitors in the management of gastroesophageal varices: a systematic review. Ann Pharmacother 2015; 49:207-219.

13 Ahmed AM, al Karawi MA, Shariq S, Mohamed AE. Frequency of gastroesophageal reflux in patients with liver cirrhosis. Hepatogastroenterology 1993; 40:478-480.

14 Zhang J, Cui PL, Lv D, Yao SW, Xu YQ, Yang ZX. Gastroesophageal reflux in cirrhotic patients without esophageal varices. World $J$ Gastroenterol 2011; 17:1753-1758.

15 de Jager CP, Wever PC, Gemen EF, van Oijen MG, van GageldonkLafeber AB, Siersema PD, et al. Proton pump inhibitor therapy predisposes to community-acquired streptococcus pneumoniae pneumonia. Aliment Pharmacol Ther 2012; 36:941-949.

16 Asim Syed IA, Abbas Naqvi SH. Proton pump inhibitors use; beware of side-effects. J Pak Med Assoc 2016; 66:1314-1318.

17 Ratelle M, Perreault S, Villeneuve JP, Tremblay L. Association between proton pump inhibitor use and spontaneous bacterial peritonitis in cirrhotic patients with ascites. Can J Gastroenterol Hepatol 2014; 28:330-334.

18 Huang KW, Kuan YC, Luo JC, Lin CL, Liang JA, Kao CH. Impact of long-term gastric acid suppression on spontaneous bacterial peritonitis in patients with advanced decompensated liver cirrhosis. Eur $J$ Intern Med 2016; 32:91-95.

19 Min YW, Lim KS, Min BH, Gwak GY, Paik YH, Choi MS, et al. Proton pump inhibitor use significantly increases the risk of spontaneous bacterial peritonitis in 1965 patients with cirrhosis and ascites: a propensity score matched cohort study. Aliment Pharmacol Ther 2014; 40:695-704.

20 Miura K, Tanaka A, Yamamoto T, Adachi M, Takikawa H. Proton pump inhibitor use is associated with spontaneous bacterial peritonitis in patients with liver cirrhosis. Intern Med 2014; 53:1037-1042.

21 de Vos M, De Vroey B, Garcia BG, Roy C, Kidd F, Henrion J, Deltenre P. Role of proton pump inhibitors in the occurrence and the prognosis of spontaneous bacterial peritonitis in cirrhotic patients with ascites. Liver Int 2013; 33:1316-1323.

22 Mandorfer M, Bota S, Schwabl P, Bucsics T, Pfisterer N, Summereder $\mathrm{C}$, et al. Proton pump inhibitor intake neither predisposes to spontaneous bacterial peritonitis or other infections nor increases mortality in patients with cirrhosis and ascites. Plos One 2014; 9:e110503.

23 Campbell MS, Obstein K, Reddy KR, Yang YX. Association between proton pump inhibitor use and spontaneous bacterial peritonitis. Dig Dis Sci 2008; 53:394-398.

24 Terg R, Casciato P, Garbe C, Cartier M, Stieben T, Mendizabal M, et al.; Study Group of Cirrhosis Complications of the Argentine Association for the Study of Liver Disease. Proton pump inhibitor therapy does not increase the incidence of spontaneous bacterial peritonitis in cirrhosis: a multicenter prospective study. J Hepatol 2015; 62:1056-1060.

25 Xu HB, Wang HD, Li CH, Ye S, Dong MS, Xia QJ, et al. Proton pump inhibitor use and risk of spontaneous bacterial peritonitis in cirrhotic patients: a systematic review and meta-analysis. Genet Mol Res 2015; 14:7490-7501.

26 Yu T, Tang Y, Jiang L, Zheng Y, Xiong W, Lin L. Proton pump inhibitor therapy and its association with spontaneous bacterial peritonitis incidence and mortality: a meta-analysis. Dig Liver Dis 2016; 48:353-359.

27 Deshpande A, Pasupuleti V, Thota P, Pant C, Mapara S, Hassan S, et al. Acid-suppressive therapy is associated with spontaneous bacterial peritonitis in cirrhotic patients: a meta-analysis. $J$ Gastroenterol Hepatol 2013; 28:235-242.

28 Khan MA, Kamal S, Khan S, Lee WM, Howden CW. Systematic review and meta-analysis of the possible association between pharmacological gastric acid suppression and spontaneous bacterial peritonitis. Eur J Gastroenterol Hepatol 2015; 27:1327-1336.
29 Tornai T, Vitalis Z, Sipeki N, Dinya T, Tornai D, Antal-Szalmas P, et al. Macrophage activation marker, soluble CD163, is an independent predictor of short-term mortality in patients with cirrhosis and bacterial infection. Liver Int 2016; 36:1628-1638.

30 D'Amico G, Garcia-Tsao G, Pagliaro L. Natural history and prognostic indicators of survival in cirrhosis: a systematic review of 118 studies. $J$ Hepatol 2006; 44:217-231.

31 Runyon BA; Practice Guidelines Committee, American Association for the Study of Liver Diseases (AASLD). Management of adult patients with ascites due to cirrhosis. Hepatology 2004; 39:841-856.

32 Dultz G, Piiper A, Zeuzem S, Kronenberger B, Waidmann O. Proton pump inhibitor treatment is associated with the severity of liver disease and increased mortality in patients with cirrhosis. Aliment Pharmacol Ther 2015; 41:459-466.

33 Parkman HP, Urbain JL, Knight LC, Brown KL, Trate DM, Miller MA, et al. Effect of gastric acid suppressants on human gastric motility. Gut 1998; 42:243-250.

34 Sanaka M, Yamamoto T, Kuyama Y. Effects of proton pump inhibitors on gastric emptying: a systematic review. Dig Dis Sci 2010; 55:2431-2440.

35 Goddard AF, Spiller RC. The effect of omeprazole on gastric juice viscosity, ph and bacterial counts. Aliment Pharmacol Ther 1996; 10:105-109.

36 Fujiwara Y, Watanabe T, Muraki M, Yamagami H, Tanigawa T, Shiba M, et al. Association between chronic use of proton pump inhibitors and small- intestinal bacterial overgrowth assessed using lactulose hydrogen breath tests. Hepatogastroenterology 2015; 62:268-272.

37 Paik CN, Choi MG, Lim CH, Park JM, Chung WC, Lee KM, et al. The role of small intestinal bacterial overgrowth in postgastrectomy patients. Neurogastroenterol Motil 2011; 23:e191-e196.

38 Su T, Lai S, Lee A, He X, Chen S. Meta-analysis: proton pump inhibitors moderately increase the risk of small intestinal bacterial overgrowth. J Gastroenterol 2018; 53:27-36.

39 Franco DL, Disbrow MB, Kahn A, Koepke LM, Harris LA, Harrison ME, et al. Duodenal aspirates for small intestine bacterial overgrowth: yield, PPIs, and outcomes after treatment at a tertiary academic medical center. Gastroenterol Res Pract 2015; 2015:971582.

40 Bajaj JS, Cox IJ, Betrapally NS, Heuman DM, Schubert ML, Ratneswaran $\mathrm{M}$, et al. Systems biology analysis of omeprazole therapy in cirrhosis demonstrates significant shifts in gut microbiota composition and function. Am J Physiol Gastrointest Liver Physiol 2014; 307:G951-G957.

41 Wallace JL, Syer S, Denou E, de Palma G, Vong L, McKnight W, et al. Proton pump inhibitors exacerbate NSAID-induced small intestinal injury by inducing dysbiosis. Gastroenterology 2011; 141:1314. e1-1322.e1.

42 Kedika RR, Souza RF, Spechler SJ. Potential anti-inflammatory effects of proton pump inhibitors: a review and discussion of the clinical implications. Dig Dis Sci 2009; 54:2312-2317.

43 Sargenti K, Prytz H, Strand A, Nilsson E, Kalaitzakis E. Healthcareassociated and nosocomial bacterial infections in cirrhosis: predictors and impact on outcome. Liver Int 2015; 35:391-400.

44 Thjodleifsson B. Treatment of acid-related diseases in the elderly with emphasis on the use of proton pump inhibitors. Drugs Aging 2002; 19:911-927.

45 Noor MT, Manoria P. Immune dysfunction in cirrhosis. J Clin Trans/ Hepatol 2017; 5:50-58.

46 Wiest R, Lawson M, Geuking M. Pathological bacterial translocation in liver cirrhosis. J Hepatol 2014; 60:197-209.

47 Llorente C, Jepsen P, Inamine T, Wang L, Bluemel S, Wang HJ, et al. Gastric acid suppression promotes alcoholic liver disease by inducing overgrowth of intestinal enterococcus. Nat Commun 2017; 8:837. 